\title{
Influence of exercise habits and physical fitness level on subjective fatigue symptoms in adolescent students
}

\author{
Shinichi Demura ${ }^{1}$, Takayoshi Yamada $^{2 *}$, Shigeru Shimada ${ }^{2}$, Masanobu Uchiyama $^{3}$ \\ ${ }^{1}$ Kanazawa University, Graduate School of Natural Science \& Technology Kakuma, Kanazawa, Japan; demuken@ed.kanazawa-u.ac.jp \\ ${ }^{2}$ Fukui National College of Technology, Department of Health \& Physical Education Geshi, Sabae, Japan; takay@fukui-nct.ac.jp \\ ${ }^{3}$ Akita Prefectural University, Research and Education Center for Comprehensive Science, Akita, Japan; uchiyama@akita-pu.ac.jp
}

Received 25 September 2010; revised 12 October 2010; accepted 18 October 2010.

\begin{abstract}
This study aimed to examine the influence of exercise habits and physical fitness level on subjective fatigue symptoms (SFS) in adolescent students. Four hundred and one healthy young male adults (age: $16.5+/-1.2 \mathrm{yr}$ ) participated in the physical fitness test of the Ministry of Education, Culture, Sports, Science and Technology in Japan and responded to the questionnaire on exercise habits and SFS. SFS questionnaire was conducted 6 times with a week interval during the physical fitness test. SFS was compared among groups with different physical fitness levels and exercise habit. Significant differences were found in difficulty with concentrated thinking, languor and low vigor, with the high physical fitness group having lower values of the three fatigue factors than the low physical fitness group regardless of affiliation with sports club and gyms, exercise frequency and time. SFS is largely affected by physical fitness level but very little by exercise habits.
\end{abstract}

Keywords: Physical Fitness; Exercise Habit; Feeling of Fatigue; Adolescence; Correlation

\section{INTRODUCTION}

Fatigue has been clarified to be useful as one health index in the daily life of adolescents [1]. The evaluation of fatigue state is important to understanding phenomena in various environments and to practicing healthy habits. The relationship between subjective fatigue symptoms and the living habits of adolescents has been examined, and it has been shown that the former is higher in students with shorter sleeping times and in those who do not eat breakfast [1-3]. Moreover, it was also reported that students with an unpleasant awakening and lower subjective health have high subjective fatigue symptoms
[2]. Many researchers have examined the relationship between subjective fatigue symptoms and the daily living habits of adolescents. Creating a regular life habit may reduce these fatigue symptoms.

Meanwhile, the relationships among life habits, health and physical fitness have been studied by many researchers, and it was shown that people who actively incorporate an exercise habit into their daily life have higher health and physical fitness levels $[4,5]$. Immobilization enhances the incidence rate of obesity and further life-style related diseases due to increasing inactivity [4]. Deterioration of health and a decrease in physical fitness lead to a decrease in activity ability in middle and old age [6] and also negatively affect daily living habits [7].

Muraki et al. [8] examined the relationship between physical fitness and mental health and reported that maximal oxygen consumption significantly related to tension, anxiety and tiredness. Namely, maintaining higher physical fitness levels may be required for maintaining mental health, because it relates to the adjustability to environmental changes. Moreover, performing exercise and sports produces a large psychological effect such as the elimination of unpleasantness, i.e., hostile feelings, tension and anxiety, and gaining feelings of exhilaration in addition to maintaining the wholesome physical condition stated above. Annesi. [9] examined the effect of moderate intensity exercise intervention for 10 weeks on mental status, and they reported that tension, anxiety, vigor and tiredness were improved by consecutive exercise. Steptoe and Cox. [10] reported that vigor and feelings of exhilaration are improved even by temporal exercise. Hence, it is suggested that performing exercise and sports is useful for maintaining healthy mental status by bringing about anxiolysis and antidepressant effects in addition to dissolving stress and building up resistance to it.

From the above, attaining vigorous exercise habits and maintaining/enhancing physical fitness are judged to be useful to relieve anxiety and tension due to environmen- 
tal changes in addition to preventing life-style related diseases. However, although anxiety and tension take place during temporal environmental changes, subjective fatigue symptoms are multiple mental symptoms and cause chronic confusion. Until now, the relationship among subjective fatigue symptoms, physical fitness and exercise habits has not been well examined.

This study aimed to examine the influence of exercise habits and physical fitness levels on subjective fatigue symptoms in adolescent students.

\section{MATERIALS AND METHODS}

\subsection{Subjects}

Four hundred and one healthy adolescent students aged 15 to 20 years who were enrolled in the National College of Technology in 2007 (age: $16.5+/-1.2$ yr, height: 171.3 +/- 5.9 cm, body-mass: 61.2 +/- $9.8 \mathrm{~kg}$ ) participated in this study. Subjects' physical characteristics were almost the same as the coeval Japanese standard values. Informed consent was obtained from each subject after a full explanation of the experimental project and its procedure. The experimental protocol in this study was approved by an inquiry committee of studies intended for humans, the Kanazawa University Health \& Sports Science Ethics Committee.

\subsection{Method of Physical Fitness Test of Ministry of Education, Culture, Sports, Science and Technology in Japan and Subjective Fatigue Symptoms Questionnaire}

Measurement items of physique and physical fitness were height, body-mass and the eight items in the physical fitness test of the Ministry of Education, Culture, Sports, Science and Technology in Japan (grip strength, sit-up, trunk anteflexion, repeated sideways jump, 20-meter shuttle run, 50-meter dash, standing long jump and handball throw). A subjective fatigue symptoms questionnaire proposed by Kobayashi et al. [11] was used. This questionnaire was developed to evaluate the fatigue of adolescent students and consists of the following six sub-scales (total 24 items): difficulties with concentrated thinking, languor, loss of vigor, loss of willingness, drowsiness and uncomfortable physical feeling. The contents of each fatigue sub-scale are as follows: concentration, thinking faculty and patience for "difficulties with concentrated thinking”; languor of whole and parts of the body for "languor"; perturbation of conversation and loss of vigor for "loss of vigor"; irritation over moving and hating of everything for "loss of willingness"; drowsiness and hoping for attitude adjustment for "drowsiness"; and asthenopia and feeling stiff in the shoulders for "uncomfortable physical feeling" [11-15]. Subjects responded the degree of feeling on the each content of questionnaire items dating back to one week from the time when the questionnaire was conducted by using the following five rating scales: no (1 point), not very (2 point), no preference (3 point), somewhat yes (4 point) and yes (5 point). In addition, they were assumed as an interval scale and used for analysis in this study.

\subsection{Procedures}

Prior to the physical fitness test and subjective fatigue symptoms questionnaire, subjects were given a substantial explanation of their contents. They were conducted in reference to the enduring rule of the Ministry of Education, Culture, Sports, Science and Technology in Japan and a survey method of Kobayashi et al. [11], respectively. They were conducted once a week using curricular class from the middle of April to the end of May by faculty in health and physical education with expert knowledge and experience. A subjective fatigue symptoms questionnaire was conducted six times in each curricular class, considering their variation during the physical fitness test period.

Exercise habits of the subjects was evaluated using a questionnaire attached to a record sheet of the above physical fitness test. Their contents were affiliation status to sports clubs and gyms (with or without affiliation) frequency of conducting exercise and sports [approximately every day (over 3 days/week), sometimes (1-2 days/week), occasionally (1-3 days/month) or fail] and duration of exercise and sports (under $30 \mathrm{~min}$, no fewer than 30 min, no more than 1 hour, no less than 1 hour, no more than 2 hours or over 2 hours). Subjects selected the most suitable content from each category. Exercise and sports were after-school activities, walking and gym lesson for maintaining/enhancing health and physical fitness, except for curricular class.

\subsection{Parameters}

Measured values of physical fitness test were transformed into scores based on the point table shown in its enduring rule. Moreover, total scores of each subject were calculated, and subjects were ranked from A to E based on the total point table. The number of students classified into ranks A through E were 35, 144, 172, 48 and 2 subjects, respectively. Subjects were divided into high (A and B) and low (D and E) physical fitness groups to examine the influence of physical fitness level and exercise habits on subjective fatigue symp- 
toms. Moreover, in each physical fitness group, subjects were divided into those with or without affiliation to sports clubs and gyms, frequency of conducting exercise and sports (high frequency: over 1-2 days/week, low frequency: under 1-3 days/month) and duration of exercise and sports (long time: over 1 hour, short time: under 1 hour). Each score of the items of the subjective fatigue symptoms questionnaire conducted six times was averaged, and the total scores in each sub-scale were calculated.

\subsection{Statistical Analysis}

The $\varphi$ coefficient was calculated to examine the relationship between exercise habits and physical fitness levels. Mean differences among each sub-scale of subjective fatigue symptoms were examined using two way analysis of variance. Tukey's HSD was used for post-hoc analysis. In addition, partial $\eta^{2}$ was calculated in each test to examine the effect size. A probability level of 0.05 was used as indicative of statistical significance and adjusted in reference to Bonferroni's method. Namely, $\alpha^{\prime}=$ $0.05 / 6$ was used.

\section{RESULTS}

Tables 1, 2 and 3 show the frequency distribution of subjects with or without affiliation to sports clubs and gyms, the frequency of conducting exercise and sports and the duration of exercise and sports in the high and low physical fitness groups and their $\varphi$ coefficients. All correlations between physical fitness and exercise habits were significant $(\varphi=0.334-0.377)$. Tables $\mathbf{4}, 5$ and $\mathbf{6}$ show two way analysis of variance, post-hoc and partial $\eta^{2}$ for subjective fatigue symptoms of subjects with or without affiliation to sports clubs and gyms, the frequency of conducting exercise and sports and the duration of exercise and sports in the high and low physical fitness groups. Significant differences were found in difficulties with concentrated thinking, languor and loss of vigor, and the high physical fitness group was lower than the low physical fitness group regardless of affiliation to sports clubs and gyms, frequency of conducting exercise and sports and duration of exercise and sports. Moreover, all differences were small (partial $\eta^{2}=$ 0.03-0.05).

\section{DISCUSSION}

Students with high physical fitness were assumed to have lower subjective fatigue symptoms, because fitness

Table 1. Correlation between physical fitness level and with or without affiliation to sports department and gym.

\begin{tabular}{lrrrr}
\hline & affiliated & not affiliated & $\varphi$ & $p$ \\
\hline High physical fitness group & 133 & 46 & 0.334 & 0.000 \\
Low physical fitness group & 18 & 32 & & \\
\hline
\end{tabular}

relates to adjustability to various environments. The present results showed that the high physical fitness group has lower subjective fatigue in difficulties with concentrated thinking, languor and loss of vigor. Muraki et al. [8] examined the relationship of maximal oxygen uptake during incremental graded cycle ergometer exercise with mood (tension, blues, anger, vigor, tiredness and confusion) and anxiety (state and attribute anxiety) measured by the Profile of Mood States (POMS) and State-Trait Anxiety Inventory (STAI) to clarify the relationship between physical fitness and mental health in adolescent males and females. The results showed that maximal oxygen uptake of adolescent males significantly and moderately related to tension, blues, tiredness, and state and attribute anxiety ( $r=-0.59$ to -0.76 ). The evaluation method of physical fitness differs between the present study and Muraki et al.'s study (total physical fitness and whole body endurance). However, subjective fatigue symptoms of the high physical fitness group in the present study showed a similar trend to the psychological characteristics of Muraki et al. Namely, it was suggested that students with higher physical fitness have low fatigue feelings because physical fitness affects them greatly.

Meanwhile, adequate exercise habits are useful for maintaining/enhancing health and physical fitness levels $[4,5]$. Also the present results showed a significant relationship between the physical fitness level and exercise habits as stated above (see Tables 1, $\mathbf{2}$ and 3). Moreover, because psychological effects such as attitude adjustments, sense of fulfillment in daily living and stress busters are expected with exercise and sports [9], students with vigorous exercise habits were inferred to have low subjective fatigue symptoms. However, subjective fatigue symptoms did not significantly differ between groups based on affiliation to sports clubs and gyms regardless of physical fitness level. More than half of the present students with affiliation to sports clubs and gyms belonged to the sports club of the National College of Technology (233/401: 57.1\%). Daley and Huffen. [16] examined the influence of exercise with low and moder-

Table 2. Correlation between physical fitness level and frequency of conducting exercise and sports.

\begin{tabular}{lrrrrr}
\hline & $\begin{array}{r}\text { high frequency: } \\
\text { over 1-2 } \\
\text { days/week }\end{array}$ & $\begin{array}{r}\text { low frequency: } \\
\text { under 1-3 } \\
\text { days/month }\end{array}$ & $\varphi$ & $p$ \\
\hline High physical fitness group & 140 & 39 & 0.377 & 0.000 \\
Low physical fitness group & 18 & 32 & & &
\end{tabular}

Table 3. Correlation between physical fitness level and conducting time of exercise and sports.

\begin{tabular}{lrrrrr}
\hline & $\begin{array}{r}\text { long time: } \\
\text { over 1 hour }\end{array}$ & $\begin{array}{r}\text { short time: } \\
\text { under 1 hour }\end{array}$ & $\varphi$ & $p$ \\
\hline High physical fitness group & 53 & 126 & -0.375 & 0.000 \\
Low physical fitness group & 37 & 13 & & & \\
\hline
\end{tabular}


Table 4. Two way analysis of variance, post-hoc and partial $\eta^{2}$ for subjective fatigue symptoms of with or without affiliation to sports department and gym in high and low.

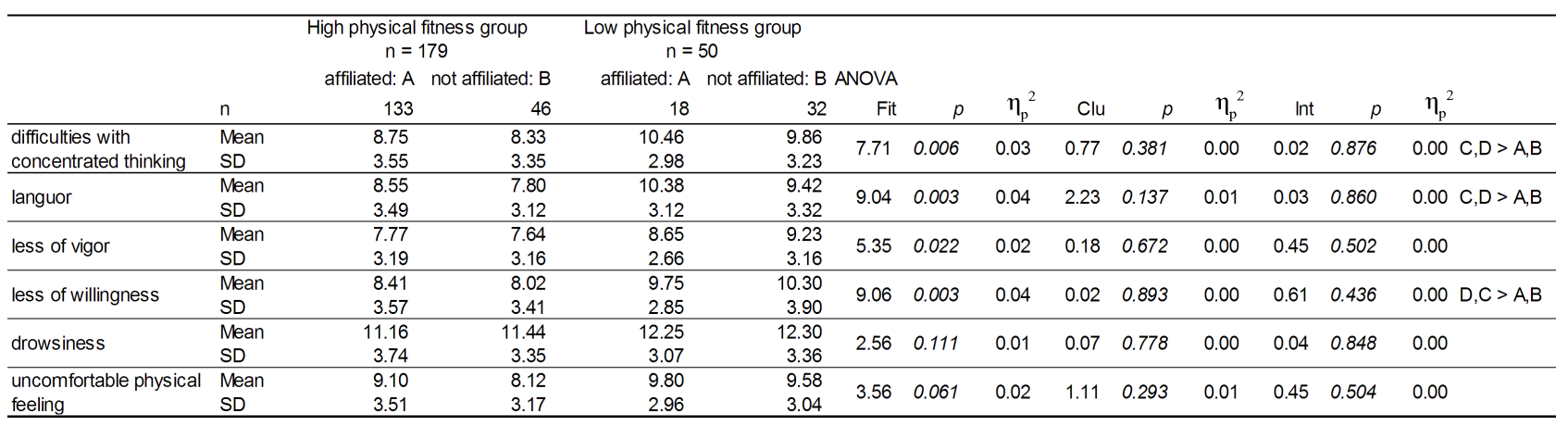

Table 5. Two way analysis of variance, post-hoc and partial $\eta^{2}$ for subjective fatigue symptoms of frequency of conducting exercise and sports in high and low physical fitness.

\begin{tabular}{|c|c|c|c|c|c|c|c|c|c|c|c|c|c|c|}
\hline & & $\begin{array}{r}\text { High physical fitn } \\
n=17 \\
\text { high frequency: } \\
\text { over } 1-2 \\
\text { davs/week }\end{array}$ & $\begin{array}{l}\text { ness group } \\
9 \\
\text { low frequency: } \\
\text { under } 1-3 \\
\text { days/month }\end{array}$ & $\begin{array}{r}\text { Low physica } \\
\text { high frequency: } \\
\text { over } 1-2 \\
\text { days/week }\end{array}$ & $\begin{array}{l}\text { al fitness group } \\
=50 \\
\text { low frequency: } \\
\text { under } 1-3 \\
\text { days } / \text { month }\end{array}$ & ANOVA & & & & & & & & \\
\hline & $\mathrm{n}$ & 140 & 39 & 18 & 32 & Fit & $p$ & $\eta_{p}^{2}$ & Fre & $p$ & $\eta_{p}^{2}$ & Int & $p$ & $\eta_{\mathrm{p}}{ }^{2}$ \\
\hline $\begin{array}{l}\text { difficulties with } \\
\text { concentrated thinking }\end{array}$ & $\begin{array}{l}\text { Mean } \\
\text { SD }\end{array}$ & $\begin{array}{l}8.73 \\
3.52 \\
\end{array}$ & $\begin{array}{l}8.33 \\
3.33 \\
\end{array}$ & $\begin{array}{r}10.34 \\
3.14 \\
\end{array}$ & $\begin{array}{l}9.93 \\
3.05 \\
\end{array}$ & 7.33 & 0.007 & 0.03 & 0.47 & 0.492 & 0.00 & 0.00 & 0.990 & $0.00 \mathrm{C}, \mathrm{D}>\mathrm{A}, \mathrm{B}$ \\
\hline languor & $\begin{array}{l}\text { Mean } \\
\text { SD }\end{array}$ & $\begin{array}{l}8.48 \\
3.47 \\
\end{array}$ & $\begin{array}{l}7.91 \\
3.08 \\
\end{array}$ & $\begin{array}{r}10.59 \\
3.06 \\
\end{array}$ & $\begin{array}{l}9.30 \\
3.21 \\
\end{array}$ & 9.02 & 0.003 & 0.04 & 2.56 & 0.111 & 0.01 & 0.38 & 0.539 & $0.00 \mathrm{C}, \mathrm{D}>\mathrm{A}, \mathrm{B}$ \\
\hline less of vigor & $\begin{array}{l}\text { Mean } \\
\text { SD }\end{array}$ & $\begin{array}{l}7.74 \\
3.24 \\
\end{array}$ & $\begin{array}{l}7.72 \\
2.88 \\
\end{array}$ & $\begin{array}{l}9.17 \\
2.77 \\
\end{array}$ & $\begin{array}{l}8.94 \\
3.04 \\
\end{array}$ & 5.94 & 0.016 & 0.03 & 0.05 & 0.820 & 0.00 & 0.03 & 0.854 & 0.00 \\
\hline less of willingness & $\begin{array}{l}\text { Mean } \\
\text { SD }\end{array}$ & $\begin{array}{l}8.29 \\
3.54 \\
\end{array}$ & $\begin{array}{l}8.36 \\
3.42 \\
\end{array}$ & $\begin{array}{r}10.17 \\
3.02 \\
\end{array}$ & $\begin{array}{r}10.07 \\
3.74 \\
\end{array}$ & 8.56 & 0.004 & 0.04 & 0.00 & 0.979 & 0.00 & 0.02 & 0.893 & $0.00 C, D>B, A$ \\
\hline drowsiness & $\begin{array}{l}\text { Mean } \\
\text { SD }\end{array}$ & $\begin{array}{r}11.23 \\
3.66 \\
\end{array}$ & $\begin{array}{r}11.25 \\
3.51 \\
\end{array}$ & $\begin{array}{r}12.25 \\
3.52 \\
\end{array}$ & $\begin{array}{r}12.30 \\
2.99 \\
\end{array}$ & 2.80 & 0.095 & 0.01 & 0.00 & 0.955 & 0.00 & 0.00 & 0.985 & 0.00 \\
\hline $\begin{array}{l}\text { uncomfortable physical } \\
\text { feeling }\end{array}$ & $\begin{array}{l}\text { Mean } \\
\text { SD }\end{array}$ & $\begin{array}{l}9.01 \\
3.46 \\
\end{array}$ & $\begin{array}{l}8.30 \\
3.27 \\
\end{array}$ & $\begin{array}{r}10.02 \\
2.63 \\
\end{array}$ & $\begin{array}{l}9.45 \\
3.10 \\
\end{array}$ & 3.49 & 0.063 & 0.02 & 1.21 & 0.274 & 0.01 & 0.02 & 0.903 & 0.00 \\
\hline
\end{tabular}

Table 6. Two way analysis of variance, post-hoc and partial $\eta^{2}$ for subjective fatigue symptoms of conducting time of exercise and sports in high and low physical fitness.

\begin{tabular}{|c|c|c|c|c|c|c|c|c|c|c|c|c|c|c|}
\hline & & \multicolumn{2}{|c|}{$\begin{array}{c}\text { High physical fitness group } \\
\text { long time: } \\
\text { short time: } \\
\text { over } 1 \text { hour } \quad \text { under } 1 \text { hour }\end{array}$} & $\begin{array}{l}\text { Low physica } \\
\text { longtime: } \\
\text { over } 1 \text { hour }\end{array}$ & $\begin{array}{l}\text { fitness group } \\
\text { short time: } \\
\text { under } 1 \text { hour }\end{array}$ & \multicolumn{2}{|c|}{ ANOVA } & \multirow[b]{2}{*}{$\eta_{0}^{2}$} & \multirow[b]{2}{*}{ Tim } & \multirow[b]{2}{*}{$p$} & \multirow[b]{2}{*}{$\eta_{p}^{2}$} & \multirow[b]{2}{*}{ Int } & \multirow[b]{2}{*}{$p$} & \multirow[b]{2}{*}{$\eta_{p}{ }^{2}$} \\
\hline & $\mathrm{n}$ & 53 & 126 & 37 & 13 & Fit & $p$ & & & & & & & \\
\hline $\begin{array}{l}\text { difficulties with } \\
\text { concentrated thinking }\end{array}$ & $\begin{array}{l}\text { Mean } \\
\text { SD }\end{array}$ & $\begin{array}{l}8.13 \\
3.36 \\
\end{array}$ & $\begin{array}{l}8.86 \\
3.51 \\
\end{array}$ & $\begin{array}{l}9.57 \\
3.16\end{array}$ & $\begin{array}{r}11.51 \\
2.34 \\
\end{array}$ & 11.14 & 0.001 & 0.05 & 4.74 & 0.030 & 0.02 & 0.97 & 0.327 & $0.00 \mathrm{D}, \mathrm{C}>\mathrm{B}, \mathrm{A}$ \\
\hline languor & $\begin{array}{l}\text { Mean } \\
\text { SD }\end{array}$ & $\begin{array}{l}7.74 \\
3.09 \\
\end{array}$ & $\begin{array}{l}8.62 \\
3.49 \\
\end{array}$ & $\begin{array}{l}9.18 \\
3.33 \\
\end{array}$ & $\begin{array}{r}11.42 \\
2.12 \\
\end{array}$ & 12.39 & 0.001 & 0.05 & 6.67 & 0.010 & 0.03 & 1.27 & 0.262 & $0.01 \mathrm{D}, \mathrm{C}>\mathrm{B}, \mathrm{A}$ \\
\hline less of vigor & $\begin{array}{l}\text { Mean } \\
\text { SD }\end{array}$ & $\begin{array}{l}7.69 \\
3.23 \\
\end{array}$ & $\begin{array}{l}7.76 \\
3.13 \\
\end{array}$ & $\begin{array}{l}8.83 \\
3.16 \\
\end{array}$ & $\begin{array}{l}9.56 \\
2.14 \\
\end{array}$ & 6.76 & 0.010 & 0.03 & 0.50 & 0.483 & 0.00 & 0.34 & 0.560 & 0.00 \\
\hline less of willingess & $\begin{array}{l}\text { Mean } \\
\text { SD }\end{array}$ & $\begin{array}{l}7.95 \\
3.38 \\
\end{array}$ & $\begin{array}{l}8.46 \\
3.56 \\
\end{array}$ & $\begin{array}{l}9.85 \\
3.86 \\
\end{array}$ & $\begin{array}{r}10.82 \\
2.01 \\
\end{array}$ & 11.15 & 0.001 & 0.05 & 1.33 & 0.250 & 0.01 & 0.13 & 0.716 & $0.00 \mathrm{D}, \mathrm{C}>\mathrm{B}, \mathrm{A}$ \\
\hline drowsiness & $\begin{array}{l}\text { Mean } \\
\text { SD }\end{array}$ & $\begin{array}{r}10.85 \\
3.62 \\
\end{array}$ & $\begin{array}{r}11.40 \\
3.62 \\
\end{array}$ & $\begin{array}{r}11.94 \\
3.35 \\
\end{array}$ & $\begin{array}{r}13.24 \\
2.47 \\
\end{array}$ & 5.25 & 0.023 & 0.02 & 2.08 & 0.150 & 0.01 & 0.34 & 0.559 & 0.00 \\
\hline $\begin{array}{l}\text { uncomfortable physical } \\
\text { feeling }\end{array}$ & $\begin{array}{l}1 \text { Mean } \\
\text { SD }\end{array}$ & $\begin{array}{l}8.14 \\
3.18 \\
\end{array}$ & $\begin{array}{l}9.15 \\
3.49 \\
\end{array}$ & $\begin{array}{l}9.36 \\
3.08 \\
\end{array}$ & $\begin{array}{r}10.51 \\
235 \\
\end{array}$ & 4.58 & 0.033 & 0.02 & 3.25 & 0.073 & 0.01 & 0.01 & 0.905 & 0.00 \\
\hline
\end{tabular}

ate intensity on perceived psychological changes and reported that, although contentment and tiredness increased significantly in the latter intensity group, no change was found in the former. Blanchard et al. [17] examined the influence of temporal exercise with 50 and $80 \%$ of maximal heart rate reserved (HRR) on perceived mood state and reported that, although no significant mood change was found in the former, soreness increased in the latter. High intensity training was likely conducted in sports clubs for enhancing performance.
Moreover, psychological characteristics that subjects perceived may have large individual differences from conducting the above stated exercise. Namely, in response to exercise intensity, some subjects feel a favorable impression, such as contentment as reported by Daley and Fuffen, and other subjects feel pain as reported by Blanchard et al. The present results may support the latter. However, as stated above, subjective fatigue symptoms did not differ between groups based on affiliation to sports clubs and gyms, and the influence of the 
physical fitness level it is judged to be greater than that of exercise habit.

Moreover, subjective fatigue symptoms did not differ in different frequencies of conducting exercise and sports (high frequency: over 1-2 days/week, low frequency: under 1-3 days/month) and duration of exercise and sports (long time: over 1 hour, short time: under 1 hour) as well as with and without affiliation to sports clubs and gyms regardless of physical fitness level. Although enhancing mental health is expected from consecutive exercise habits, 10 weeks have been reported to be required at the least [18-21]. Meanwhile, Pertruzzello et al. [18] examined exercise time using meta-analysis and reported that 21 minutes of exercise is required to reduce anxiety. Moreover, some researchers insist on the necessity to assure exercise for 20 minutes based on the above stated findings [19,22]. However, Petruzzello and Landers. [23] compared the psychological state after 15 and 30 minutes of running with moderate exercise intensity (75\% maximal oxygen uptake) and reported that a significant difference was not found between both conditions. In another similar research project, it was reported that an increase of feelings of exhilaration, and a decrease of anxiety was found by conducting five minutes of exercise [24]. More subjects in this study customarily conducted exercise more than 3days/week (150/401: 37.4\%). Moreover, $73.0 \%$ of them exceeded the exercise time needed to obtain the effect of mental health improvement proposed by the above previous studies (over 30 minutes). In addition, many of them conducted training with high intensity for enhancing performance and skills. Therefore, the present results may differ from those in the previous studies which aimed to improve mental health and examined the effect of fitness conditioning using intensity and exercise time. However, from the present results, it is inferred that the influence of physical fitness on subjective fatigue symptoms is larger than that of the frequency of conducting exercise and sports and the duration of exercise and sports.

\section{CONCLUSIONS}

In conclusion, subjective fatigue symptoms are lower in students with a higher physical fitness level than that of students with a lower physical fitness level. They are largely affected by physical fitness level but little by exercise habit.

\section{ACKNOWLEDGMENTS}

The authors have no financial disclosures to make on this paper. We contributed to all aspects of this paper and there is no sponsor to this study.

\section{REFERENCES}

[1] Kadota, S. (1990) Relationship between subjective symptoms of fatigue and living behavior in senior high school students - A study by quantification method II. Japanese Journal of School Health, 32, 239-247.

[2] Kadota, S. (1978) Examination of students' health care administration: Living conditions and subjective fatigue symptoms. Japanese Journal of School Health, 20, 286-291.

[3] Takakura, M. (1993) Relationship between type a behavior pattern and fatigue symptoms, life style in university students. Japanese Journal of School Health, 35, 484-491.

[4] Momose, Y. and Une, H. (1998) Relationship of percent body fat with living and dietary habit in adolescent students. Japanese Journal of School Health, 40, 150-158.

[5] Naka, H. and Demura, S. (1994) Influence of habitual exercise on physique and physical fitness in adolescent male students: From an examination of three-year longitudinal data. Japanese Journal of Physical Education, 39, 287-303.

[6] Shibata, H. (1987) Measurement and evaluation of the elderly's physical fitness. Journal of Health, 37, 658-661.

[7] Kimura, M. (1991) Exercise stress for the eldrly, physical fitness changes with age and exercise habit. Japanese Journal of Sports Science, 10, 722-728.

[8] Muraki, S., Maehara, T., Ishi, K., Ajimoto, M. and Kikuchi, K. (1993) Gender difference in the relationship between physical fitness and mental health. The Annals of Physiological Anthropology, 12, 379-384.

[9] Annesi, J.J. (2004) Mood states of formerly sedentary younger and older women at weeks 1 and 10 of a moderate exercise program. Psychological Reports, 94, 1337-1342.

[10] Steptoe, A. and Cox, S. (1988) Acute effects of aerobic exercise on mood. Health Psychology, 7, 329-340. doi:10.1037/0278-6133.7.4.329

[11] Kobayashi, H., Demura, S., Goshi, F., Sato, S. and Noda, M. (2000) Construction of a subjective fatigue scale for adolescent students. Japanese Journal of Public Health, 47, 638-645.

[12] Demura, S., Kobayashi, H. and Matsuzawa, J. (1997) Examination of proposed items for a fatigue questionnaire for students based on subjective symptoms. Japanese Journal of Public Health, 44, 427-439.

[13] Kobayashi, H., Demura, S. and Goshi, F. (1998) Sex differences of fatigue in young men and women-From the view point of experience of awareness and cognitive appraisal-. Japanese Journal of Physical Fitness and Sports Medicine, 47, 581-592.

[14] Demura, S., Kobayashi, H. and Sato, S. (2000) Examination of validity of the subjective fatigue scale for young adults. Japanese Journal of Public Health, 48, 76-84.

[15] Kobayashi, H., Demura, S. and Sato, S. (2001) Relationship between a subjective fatigue scale for young adults and the subjective symptoms index Japanese. Journal of Physical Education, 46, 35-46.

[16] Daley, A.J. and Huffen, C. (2003) The effects of low and moderate intensity exercise on subjective experiences in a naturalistic health and fitness club setting. Journal of Health Psychology, 8, 685-691. 
doi:10.1177/13591053030086003

[17] Blanchard, C.M., Rodgers, W.M., Spence, J.C. and Courneya, K.S. (2001) Feeling state responses to acute exercise of high and low intensity. Journal of Science and Medicine in Sport, 4, 30-38. doi:10.1016/S1440-2440(01)80005-0

[18] Petruzzello, S.J., Landers, D.M., Hatield, B.D., Kubitz, K.A. and Salazar, W. (1991) A meta-analysis on the anxiety-reducing effects of acute and chronic exercise. Sports Medicine, 11, 143-182. doi:10.2165/00007256-199111030-00002

[19] Berger, B.G. and Owen, D.R. (1987) Anxiety reduction with swimming: Relationships between exercise and state, trait, and somatic anxiety. International Journal of Sports Psychology, 18, 286-302.

[20] Doyne, E.J., Ossip-Klein, D.J., Bowman, E.D., Osborn, K.M., McDowgall-Wilson, I.B. and Neimeyer, R.A.
(1987) Running versus weight lifting in the treatment of depression. Journal of Consulting and Clinical Psychology, 55, 748-754. doi:10.1037/0022-006X.55.5.748

[21] LaFontaine, T. (1999) Long-term effects of aerobic exercise on psychological outcomes. Preventive Medicine, 28, 75-85. doi:10.1006/pmed.1998.0385

[22] Leith, L.M. (1994) Foundation of exercise and mental health. Fitness Information Technology, West Virginia Morgantown.

[23] Petruzzello, S.J. and. Landers, D.M (1995) Varying the duration of acute exercise: Implications for changes in affect. Journal of Sports Medicine and Physical Fitness, 35, 295-302.

[24] Hashimoto, K., Saitou, A., Tokunaga, K., Hanamura, S. and Isogai, H. (1996) Feeling changes during running with comfortable self-pace and the recovery. Japanese Journal of Physical Education, 10, 31-40. 